\title{
MISDIAGNOSIS OF HEREDITARY AMYLOIDOSIS AS AL (PRIMARY) AMYLOIDOSIS
}

\author{
Helen J. Lachmann, M.B., B.ChiR., David R. Booth, Ph.D., Susanne E. Booth, Alison Bybee, Ph.D., \\ Janet A. Gilbertson, Julian D. Gillmore, M.B., B.S., M.D., Mark B. Pepys, M.D., Ph.D., \\ and Philip N. Hawkins, M.B., B.S., Ph.D.
}

\begin{abstract}
Background Hereditary, autosomal dominant amyloidosis, caused by mutations in the genes encoding transthyretin, fibrinogen A $\alpha$-chain, lysozyme, or apolipoprotein A-I, is thought to be extremely rare and is not routinely included in the differential diagnosis of systemic amyloidosis unless there is a family history.

Methods We studied 350 patients with systemic amyloidosis, in whom a diagnosis of the light-chain $(A L)$ type of the disorder had been suggested by clinical and laboratory findings and by the absence of a family history, to assess whether they had amyloidogenic mutations.

Results Amyloidogenic mutations were present in 34 of the 350 patients (9.7 percent), most often in the genes encoding fibrinogen A $\alpha$-chain (18 patients) and transthyretin (13 patients). In all 34 of these patients, the diagnosis of hereditary amyloidosis was confirmed by additional investigations. A low-grade monoclonal gammopathy was detected in 8 of the 34 patients (24 percent).

Conclusions A genetic cause should be sought in all patients with amyloidosis that is not the reactive systemic amyloid A type and in whom confirmation of the AL type cannot be obtained. (N Engl J Med 2002; 346:1786-91.)

Copyright (c) 2002 Massachusetts Medical Society.
\end{abstract}

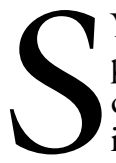
YSTEMIC amyloidosis is the diagnosis in 2.5 percent of all native renal biopsies, ${ }^{1}$ and the cause of death in more than 1 in 1500 people in the United Kingdom annually. Acquired monoclonal immunoglobulin light-chain (AL) amyloidosis, formerly known as primary amyloidosis, is the most common form of systemic amyloidosis and can respond to chemotherapy directed at the underlying plasma-cell dyscrasia. ${ }^{2-5}$ Scintigraphy with labeled serum amyloid P component (SAP), a technique for quantitatively imaging amyloid deposits in vivo, ${ }^{6}$ has shown that deposits frequently regress after a reduction in monoclonal light-chain production. ${ }^{7}$ The chief consideration in the differential diagnosis of AL amyloidosis is reactive systemic amyloid A (AA) amyloidosis, but this form of the disorder is always a complication of chronic inflammation, and amyloid A deposits can usually be verified immunohistochemically. ${ }^{8}$ Another possibility is hereditary systemic amy- loidosis, in which the amyloid fibrils are usually derived from genetic variants of transthyretin, ${ }^{9}$ apolipoprotein A-I, ${ }^{10,11}$ lysozyme, ${ }^{12}$ or fibrinogen A $\alpha$-chain. ${ }^{13}$ However, these autosomal dominant conditions, which are thought to be extremely rare, are generally not considered in the differential diagnosis in patients without a relevant family history.

The diagnosis of hereditary amyloidosis has implications for prognosis, genetic counseling, and treatment, which may include liver transplantation to correct the underlying metabolic defect. Although most reported mutations causing hereditary amyloidosis show high penetrance, amyloidogenic mutations have occasionally been identified in asymptomatic elderly patients. In addition, population and haplotype studies have raised the possibility that two specific mutations associated with hereditary amyloidosis - one encoding the substitution of methionine for valine at position 30 of transthyretin (Val30Met) and one encoding the substitution of valine for glutamic acid at position $\mathbf{5 2 6}$ of fibrinogen A $\alpha$-chain (Glu526Val) - may not be rare. ${ }^{14}$ We systematically studied patients with apparently sporadic systemic AL amyloidosis to determine whether any of the patients in fact had hereditary amyloidosis.

\section{METHODS}

\section{Patients}

The genes for transthyretin, apolipoprotein A-I, lysozyme, and fibrinogen A $\alpha$-chain were studied in all 350 patients referred to the National Amyloidosis Centre of the United Kingdom between 1997 and 2000 with biopsy-proved amyloidosis and a presumptive diagnosis of systemic AL amyloidosis. All the patients gave oral informed consent for the genetic analyses. None of them were aware of any illness in their family that was consistent with the presence of hereditary amyloidosis, and the amyloid in each case was shown by immunohistochemical analysis not to be the AA type. Serum and urine electrophoresis and immunofixation showed that 80 percent of the patients had a monoclonal gammopathy, a proportion similar to those in other series of patients with AL amyloidosis. ${ }^{15}$

In all the patients, clinical assessment included electrocardiography, Doppler echocardiography, and scintigraphy with ${ }^{123}$ I-labeled SAP, a method of imaging visceral amyloid deposits. Comprehensive immunohistochemical staining for amyloid fibril proteins was

From the National Amyloidosis Centre and Centre for Amyloidosis and Acute Phase Proteins, Department of Medicine, Royal Free and University College Medical School, Royal Free Campus, London. Address reprint requests to Dr. Hawkins at the National Amyloidosis Centre, Department of Medicine, Royal Free and University College Medical School, Royal Free Campus, Rowland Hill St., London NW3 2PF, United Kingdom, or at p.n.hawkins@rfc.ucl.ac.uk. 
performed on amyloid-containing tissue from patients in whom potentially amyloidogenic mutations were identified. In addition, newly identified mutations in the genes for amyloid proteins were sought in 50 anonymous white controls from the general British population. Twenty-two clinically healthy first-degree relatives of patients with hereditary fibrinogen A $\alpha$-chain Glu526Val amyloidosis were also studied.

\section{Genotyping, Scintigraphy, and Immunohistochemistry}

DNA was isolated as previously described from samples of whole blood treated with EDTA. ${ }^{11}$ The coding regions of the genes encoding transthyretin, apolipoprotein A-I, fibrinogen A $\alpha$-chain (part of exon 5 ), and lysozyme (exon 2) were amplified by the polymerasechain-reaction assay with the use of primers, solutions, and cycling conditions that have been described elsewhere.11,12,16,17 The products of the polymerase chain reaction were sequenced with the use of terminator reagent (ABI BigDye version 3.0, AB Applied Biosystems) according to the manufacturer's instructions.

Anterior and posterior whole-body scintigrams (Elscint Super Helix, GE Medical Systems) were obtained 24 hours after the intravenous injection of $200 \mathrm{MBq}$ of ${ }^{123}$ I-labeled SAP. ${ }^{6}$ The scans were interpreted by a single physician with extensive experience.

Six-micrometer tissue sections were tested for the presence of amyloid by Congo-red staining and a search for pathognomonic red-green birefringence under cross-polarized light microscopy. ${ }^{18}$ Immunohistochemical staining was performed on $2-\mu \mathrm{m}$ sections of amyloid-containing tissue with the use of commercial antiserum (Dako; Medix; and Helena Biosciences) against serum amyloid A protein, immunoglobulin $\kappa$ and $\lambda$ light chains, transthyretin, lysozyme, apolipoprotein A-I, and fibrinogen, as previously described. ${ }^{19}$ Positive control tissues containing each of these types of amyloid protein were also stained during each run.

\section{RESULTS}

Of the 350 patients with systemic amyloidosis in this study, 18 (5.1 percent) were heterozygous for a point mutation that encoded the Glu526Val substitution in fibrinogen A $\alpha$-chain. This mutation was not present in the 50 healthy controls. All 18 patients were of northern European ancestry, and none were aware of any relevant family history. However, genealogic studies performed after the genetic diagnosis revealed that two of the patients were cousins and that ancestors of two other patients had lived in adjacent villages. A fifth patient retrospectively ascertained that her dizygotic twin had died of renal failure at the age of 76 years. The other 13 patients had no such history and apparently were not related. The median age of the 18 patients at the time of presentation was 59 years; the youngest was in her $30 \mathrm{~s}$, and the oldest was 78 years old. At this writing, the latter patient remains well at the age of 81 , despite impaired renal function. All 18 patients presented with isolated renal dysfunction and proteinuria, and most of them had moderate hypertension. Spontaneous splenic rupture occurred in two patients. Twelve patients became dependent on dialysis, with a median interval of 2.3 years between presentation and the development of renal failure. All nine of the patients who were followed up for more than five years eventually had end-stage renal failure.

The distribution of fibrinogen A $\alpha$-chain amyloid revealed by ${ }^{123}$ I-labeled SAP scintigraphy was consistent. All 18 patients with the Glu526Val variant had renal deposits, and splenic amyloid was present in all but 1 . Two patients, both of whom had initially presented with renal impairment more than a decade before their participation in this study, had hepatic amyloidosis. Amyloid deposits in bone, a pathognomonic feature of AL amyloidosis, were not identified in any of the patients. The electrocardiogram and echocardiogram did not suggest the presence of cardiac amyloidosis in any of the patients, and none had evidence of peripheral or autonomic neuropathy. In addition, none of the patients had clinical evidence of hemorrhagic or thrombotic tendencies, and the thrombin time, prothrombin time, activated partialthromboplastin time, and fibrinogen levels were normal in all cases. Four of the 18 patients with a mutation in the gene encoding fibrinogen $\mathrm{A} \alpha$-chain also had low-grade paraproteinemia; in each case the level of paraprotein was less than $0.2 \mathrm{~g}$ per deciliter. The presence of these monoclonal gammopathies had reinforced the initial misdiagnosis of AL amyloidosis, and three of the four patients had received cytotoxic chemotherapy without a clinical response (Fig. 1).

Twelve of the 22 first-degree relatives of patients with fibrinogen A $\alpha$-chain amyloidosis were heterozygous for the mutation encoding the Glu526Val variant. All of them were more than 50 years old, and none had proteinuria or evidence of amyloidosis on 123I-labeled SAP scintigraphy.

Thirteen patients were heterozygous for point mutations in the gene encoding transthyretin (Table 1); three of these point mutations have apparently not been described previously: Phe33Val, Asp38Val, and Alal20Ser (GenBank accession numbers AF485254, AF485253, and AF485252, respectively). All 13 of these patients presented with cardiac amyloidosis and variable degrees of autonomic and peripheral neuropathy. In none of them did ${ }^{123}$ I-labeled SAP scintigraphy reveal any amyloid deposits in the liver or bone; such deposits have not been noted in transthyretin-associated amyloidosis. None of these 13 patients had any relevant family history.

One patient who had presented with slowly progressive renal impairment was heterozygous for an amyloidogenic mutation encoding a lysozyme variant (in which histidine replaces aspartic acid at position 67 [Asp67His]), and another patient was heterozygous for a mutation encoding an apolipoprotein A-I variant (in which arginine replaces glycine at position 26 [Gly26Arg]). A man who had presented with hoarseness due to laryngeal amyloid and a long history of sterility with testicular amyloid on biopsy and in whom ${ }^{123}$ I-labeled SAP scintigraphy showed subclinical renal deposits of amyloid was found to be heterozygous for a novel mutation encoding an ap- 


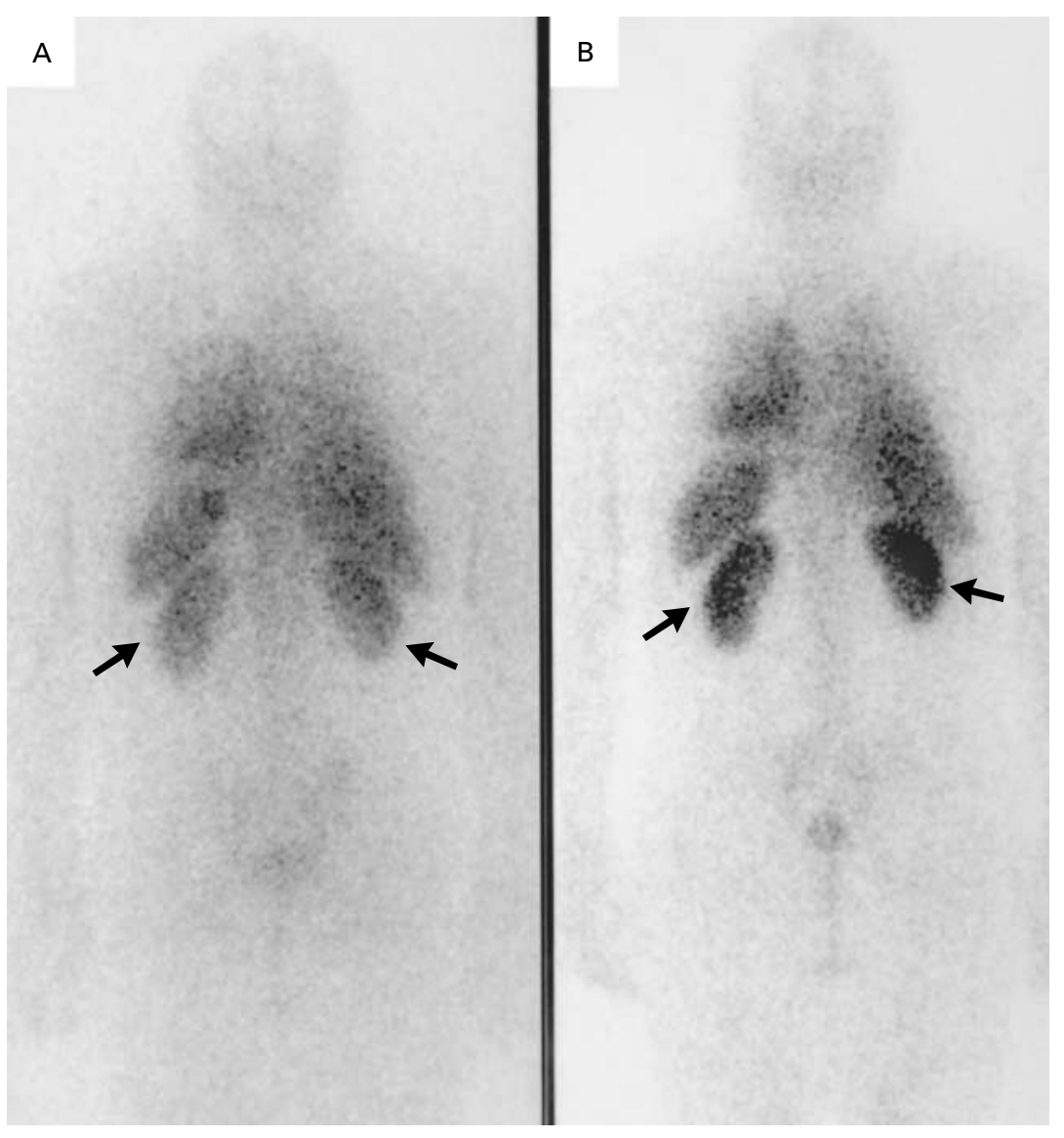

Figure 1. Progression of Amyloid Deposits in a Patient with the Glu526Val Variant of Fibrinogen A $\alpha$-Chain Amyloidosis and an Incidental Monoclonal Gammopathy.

Serial posterior whole-body scintigraphic images were obtained after the intravenous injection of 123|-labeled serum amyloid P component in a 48-year-old patient who was thought to have AL amyloidosis and who received high-dose chemotherapy, with complete resolution of his monoclonal gammopathy. The scan at the time of diagnosis (Panel A) shows a moderate degree of abnormal uptake into renal amyloid deposits (arrows). The degree of uptake had increased substantially at the time of a follow-up examination three years later (arrows in Panel B).

olipoprotein A-I variant (in which proline replaces alanine at position 175 [Alal75Pro]) (GenBank accession number, AF485255).

Renal-biopsy specimens were available from 17 of the patients with the Glu526Val variant of fibrinogen A $\alpha$-chain (Fig. 2). In all specimens, the amyloid deposits stained specifically with antifibrinogen antibodies, but there was marked variation in the intensity of the staining. There was staining for lysozyme and apolipoprotein A-I in specimens from the 3 patients who had mutations in the corresponding genes and staining for transthyretin in each of the 13 patients with a transthyretin variant. Robust, reproducible, immunospecific staining of amyloid deposits composed of these variant proteins required extensive preparation of the tissue specimens with the use of methods that included trypsin and microwave pretreatment.

Intact monoclonal immunoglobulins were detected in the serum of 8 of the 34 patients with hereditary amyloidosis (24 percent), at levels of less than $0.2 \mathrm{~g}$ per deciliter, but in none of these patients were free light chains identified in the urine. By comparison, circulating paraproteins or urinary free light chains were present in 273 of the remaining 316 patients with $\mathrm{AL}$ amyloidosis (86 percent). However, there was no specific immunohistochemical staining of amyloid deposits with antibodies to $\kappa$ or $\lambda$ immunoglobulin light chains in any of the patients with hereditary amyloidosis or in 195 of the 316 patients with AL amyloidosis (62 percent). 
Table 1. Characteristics of 16 Patients with Hereditary Amyloidosis Due to a Mutation in the Gene Encoding Transthyretin, Lysozyme, or Apolipoprotein A-I.

\begin{tabular}{|c|c|c|c|c|c|}
\hline Patient No. & Protein and Mutation & $\begin{array}{c}\text { Age at } \\
\text { Presentation (Yr) }\end{array}$ & Predominant Clinical Features & $\begin{array}{c}\text { EthNic or } \\
\text { National Origin* }\end{array}$ & $\begin{array}{l}\text { Sites of AMYLOID } \\
\text { INVOLVEMENTt }\end{array}$ \\
\hline & Transthyretin & & & & \\
\hline 1 & Val30Met & 62 & Neuropathy & Irish & Heart, kidneys \\
\hline 2 & Phe33Val & 39 & Neuropathy & English & Heart, spleen, kidneys \\
\hline 3 & Phe33Leu & 57 & Cardiomyopathy, neuropathy & Polish & Heart, kidneys \\
\hline 4 & Asp38Val & 58 & Neuropathy & Ghanaian & Heart, spleen \\
\hline 5 & Gly47Glu & 45 & $\begin{array}{l}\text { Cardiomyopathy, neuropathy, } \\
\text { nephropathy }\end{array}$ & English & Heart, spleen, kidneys \\
\hline 6 & Thr60Ala & 54 & Cardiomyopathy, neuropathy & Scottish & Heart, kidneys \\
\hline 7 & Thr60Ala & 65 & Neuropathy & English & Heart, spleen \\
\hline 8 & Thr60Ala & 73 & Cardiomyopathy & Irish & Heart, spleen, kidneys \\
\hline 9 & Thr60Ala & 67 & Neuropathy & Irish & Heart \\
\hline 10 & Thr60Ala & 63 & Cardiomyopathy, neuropathy & Irish & Heart \\
\hline 11 & Alal20Ser & 62 & Cardiomyopathy, neuropathy & Afro-Caribbean & Heart, spleen \\
\hline 12 & Vall22Ile & 74 & Cardiomyopathy & Afro-Caribbean & Heart \\
\hline 13 & Val122Ile & 63 & Cardiomyopathy, neuropathy & Afro-Caribbean & Heart \\
\hline 14 & $\begin{array}{l}\text { Lysozyme } \\
\text { Asp67His } \\
\text { Apolipoprotein A-I }\end{array}$ & 58 & Nephropathy & English & Spleen, kidneys \\
\hline 15 & Gly26Arg & 28 & Nephropathy & Irish & Liver, spleen, kidneys \\
\hline 16 & Alal75Pro & 35 & Hoarseness, sterility & English & Kidneys \\
\hline
\end{tabular}

*Information on ethnic or national origin was provided by the patients.

†Sites of amyloid involvement were assessed by echocardiography and scintigraphy with ${ }^{123}$ I-labeled serum amyloid P component.

\section{DISCUSSION}

The identification of hereditary amyloidosis in almost 10 percent of patients with a presumptive diagnosis of systemic AL amyloidosis has several clinical implications. The single-gene mutations that cause hereditary amyloidosis evidently have variable penetrance, and most patients who have amyloidosis associated with the fibrinogen A $\alpha$-chain Glu526Val variant - the most common form of hereditary amyloidosis identified in this study - do not have a relevant family history. Therefore, we now routinely perform DNA analysis in all patients with systemic amyloidosis. This practice has already prevented the inappropriate administration of chemotherapy to patients with a presumptive diagnosis of AL disease and has enabled potentially curative liver transplantations to be performed in four patients with previously unsuspected hereditary amyloidosis.

The clinical manifestations of the AL type of systemic amyloidosis are extremely heterogeneous. Macroglossia and periorbital ecchymoses are virtually pathognomonic, but they occur in only a minority of cases. Indeed, some of the characteristic patterns of organ involvement in AL amyloidosis are indistinguishable from those in familial amyloid polyneuropathy and hereditary non-neuropathic systemic amyloidosis. All patients with $\mathrm{AL}$ amyloidosis have an underlying clonal B-cell dyscrasia, but in only about 85 percent can a monoclonal protein be detected in the serum or urine. Because subtle monoclonal gammopathies are not in- frequent in the general population, ${ }^{20}$ the detection of paraprotein in a patient with amyloidosis may be misleading, and thus it is essential to identify the actual amyloid fibril protein by immunohistochemical analysis whenever possible. Immunohistochemistry is usually definitive in identifying or ruling out AA amyloidosis, but it frequently is not diagnostic with respect to AL amyloidosis. ${ }^{8,21}$ In our series, AL fibrils were identified by immunohistochemical staining in only 121 of the 316 patients with confirmed AL disease (38 percent). This reflects the failure of anti-light-chain antibodies to bind to light-chain fragments with an abnormal cross $-\beta$ amyloid-fibril conformation and also reflects background staining of normal immunoglobulins in the tissues. Specific fixation procedures or the use of unfixed, fresh-frozen tissue may yield better results, but ideally processed material is often not available after routine biopsies. Although the stored biopsy material we studied had not been uniformly processed, extensive use of optimization techniques allowed immunohistochemical confirmation of the DNA findings in each case.

The most common of the autosomal dominant systemic amyloidoses is caused by transthyretin variants. Patients usually present with familial amyloid polyneuropathy, with progressive peripheral and autonomic neuropathy; involvement of the heart or kidneys is variable. More than 80 amyloidogenic mutations in the gene encoding transthyretin have been identified, ${ }^{22}$ and we found 3 hitherto unreported variants. We also 
detected a transthyretin variant in which isoleucine replaces valine at position 122 (Vall22Ile) in two patients, one of whom had amyloid neuropathy and cardiomyopathy. This variant occurs in 4 percent of black Americans, ${ }^{23}$ in whom it usually is silent or is associated with isolated late-onset cardiac amyloidosis. A transthyretin variant in which alanine replaces threonine at position 60 (Thr60Ala) is also associated with lateonset disease, reducing the likelihood of a relevant family history.

Patients with hereditary non-neuropathic systemic amyloidosis, which is caused by mutations in the genes encoding lysozyme, ${ }^{12}$ apolipoprotein A- ${ }^{10,11}$ or fibrinogen $\mathrm{A} \alpha$-chain, ${ }^{13}$ usually present with renal dysfunction. A mutation in the gene encoding apolipoprotein A-II ${ }^{24}$ has recently also been associated with hereditary renal amyloidosis. However, fewer than 30 families affected by any of these mutations have been described. The Glu526Val variant of the fibrinogen A $\alpha$-chain ${ }^{25}$ has been reported in five families, all with clear cases of autosomal dominant amyloidosis. The present finding that the underlying mutation has low penetrance explains the previously enigmatic finding that haplotype studies in four of the families suggested that they had a common ancestor. ${ }^{26}$

The distinctive clinical picture of amyloidosis associated with the Glu526Val variant of fibrinogen A $\alpha$-chain may reflect the tropism of this amyloid variant for the kidneys and its remarkable selectivity for glomeruli. All our patients presented with apparently isolated renal dysfunction, and in contrast to the usual progression of AL amyloidosis, the course of illness was prolonged and relatively benign. In most of the patients, renal dysfunction occurred after the age of 50 years, but in all of them end-stage renal failure associated with progressive accumulation of amyloid occurred within five years. The decline of renal function may have been exacerbated by hypertension in some of the patients, but no other risk factors for renal dysfunction were identified. In two of the patients, transplanted kidneys failed within six years because of severe amyloidosis in the graft. Only one patient had clinically

Figure 2. Renal-Biopsy Specimen from a Patient with the Glu526Val Variant of Fibrinogen A $\alpha$-Chain Amyloidosis.

Panel A shows that the glomeruli are strikingly enlarged and that the normal architecture is almost entirely obliterated by amyloid deposition; the vessels and tubular interstitium, in contrast, contain remarkably little amyloid (Congo red stain, $\times 100$ ). In Panel $B$, the same section viewed under cross-polarized light shows apple-green birefringence $(\times 100)$. In Panel C, immunohistochemical staining with polyclonal sheep antifibrinogen antibodies confirms the presence of fibrinogen within the deposits $(\times 100)$.

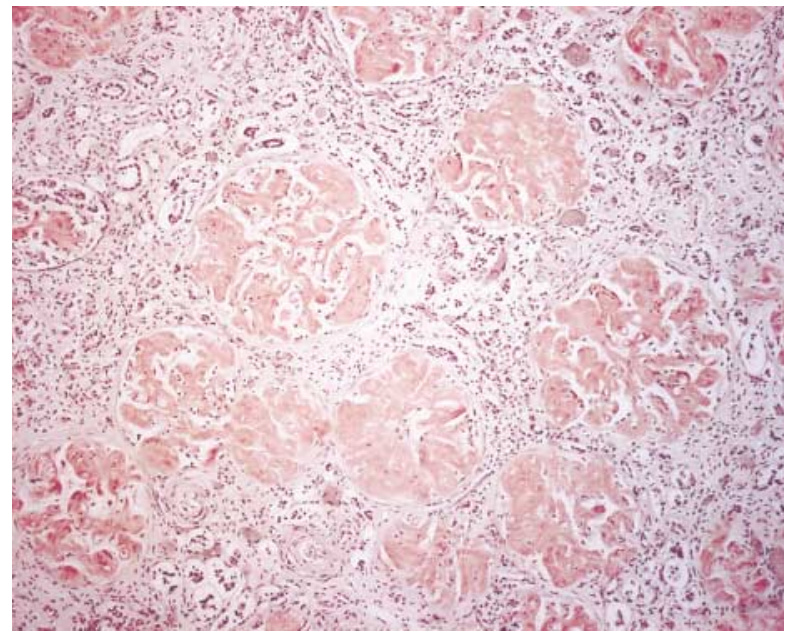

A

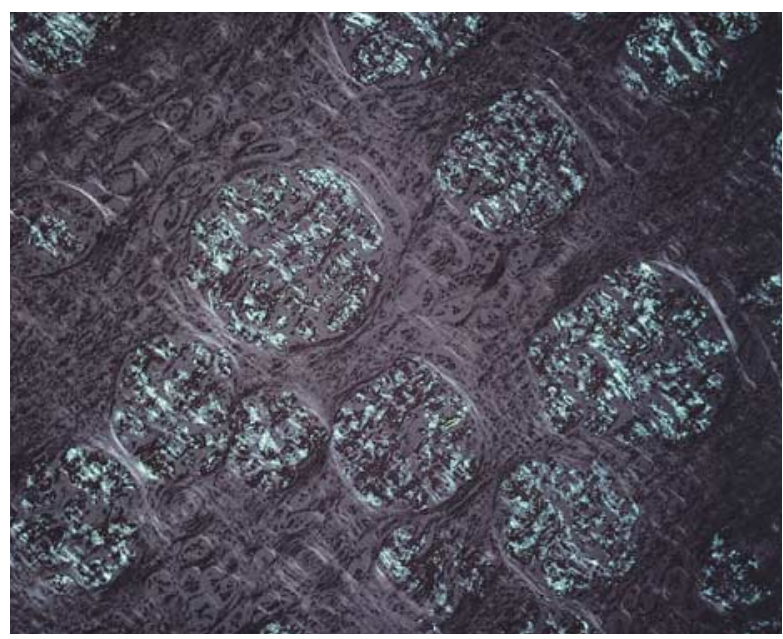

B

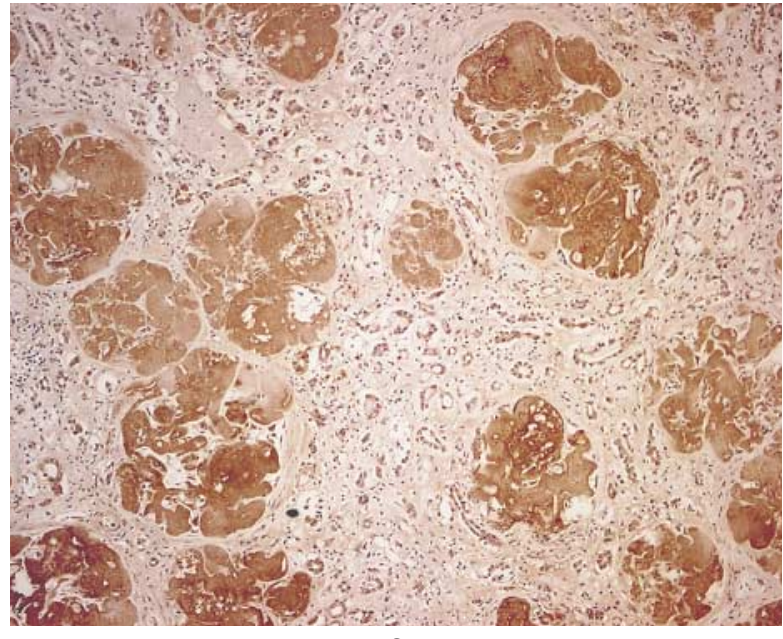

$\mathrm{C}$ 
significant extrarenal disease, in the form of liver failure, 15 years after her presentation. Another patient had subclinical deposition of amyloid in his liver 13 years after his kidneys had failed, and it is likely that the hepatic deposition was a late manifestation of this mutation. In this type of hereditary amyloidosis, unlike AL amyloidosis, cardiac involvement is not a feature.

In the patients with amyloidosis caused by the Asp67His variant of lysozyme and the Gly26Arg variant of apolipoprotein A-I, progressive renal impairment developed slowly, a phenotype similar to that in previously described kindreds. ${ }^{27,28}$ The patient with the Alal75Pro variant of apolipoprotein A-I, a newly identified variant, had hoarseness due to laryngeal amyloid deposits, a feature that commonly occurs in localized AL amyloidosis and that has also been reported in patients with mutations that disrupt this region of the apolipoprotein A-I molecule. ${ }^{29,30}$

AL amyloidosis often responds to chemotherapy that suppresses the underlying clonal plasma-cell disorder, ${ }^{2-5}$ but chemotherapy has no role in the treatment of hereditary amyloidosis and is dangerous. The types of hereditary amyloidosis in which the amyloidogenic protein is synthesized solely by the liver can be effectively treated by liver transplantation. This form of "surgical gene therapy" 31 has been successful in familial amyloid polyneuropathy associated with variant forms of transthyretin and in amyloidosis due to the Glu526Val variant of fibrinogen A $\alpha$-chain. ${ }^{17}$ However, the rate of progression of hereditary amyloidosis caused by variants of lysozyme, apolipoprotein $\mathrm{A}-\mathrm{I}$, and fibrinogen $\mathrm{A} \alpha$-chain is slow in many patients, in whom supportive measures and kidney transplantation alone are associated with an excellent outcome.

Supported in part by grants from the Medical Research Council, United Kingdom, and the Wellcome Trust (to Dr. Pepys and Dr. Hawkins); by a Wellcome Trust Research Training Fellowship (to Dr. Gillmore); and by National Health Service Research and Development Funds.

We are indebted to our many colleagues for referring and caring for the patients; to Sheril Madhoo, Dorothea Gopaul, and Jayshree Joshi for participating in the care and study of the patients at the National Amyloidosis Centre; and to Beth Jones for assistance in the preparation of the manuscript.

\section{REFERENCES}

1. Davison AM. The United Kingdom Medical Research Council's glomerulonephritis registry. Contrib Nephrol 1985;48:24-35.

2. Gillmore JD, Davies J, Iqbal A, Madhoo S, Russell NH, Hawkins PN. Allogeneic bone marrow transplantation for systemic AL amyloidosis. $\mathrm{Br} \mathrm{J}$ Haematol 1998;100:226-8.

3. Kyle RA, Gertz MA, Greipp PR, et al. A trial of three regimens for primary amyloidosis: colchicine alone, melphalan and prednisone, and melphalan, prednisone, and colchicine. N Engl J Med 1997;336:1202-7. 4. Wardley AM, Jayson GC, Goldsmith DJ, Venning MC, Ackrill P, Scarffe $\mathrm{JH}$. The treatment of nephrotic syndrome caused by primary (light chain) amyloid with vincristine, doxorubicin and dexamethasone. Br J Cancer 1998;78:774-6.

5. Gertz MA, Lacy MQ, Dispenzieri A. Myeloablative chemotherapy with stem cell rescue for the treatment of primary systemic amyloidosis: a status report. Bone Marrow Transplant 2000;25:465-70.

6. Hawkins PN, Lavender JP, Pepys MB. Evaluation of systemic amyloidosis by scintigraphy with ${ }^{123}$ I-labeled serum amyloid P component. N Engl J Med 1990;323:508-13.

7. Hawkins PN. Studies with radiolabelled serum amyloid P component provide evidence for turnover and regression of amyloid deposits in vivo. Clin Sci (Lond) 1994;87:289-95.

8. Tan SY, Pepys MB. Amyloidosis. Histopathology 1994;25:403-14.

9. Benson MD, Uemichi T. Transthyretin amyloidosis. Amyloid 1996;3: 44-56.

10. Nichols WC, Dwulet FE, Liepnieks J, Benson MD. Variant apolipoprotein AI as a major constituent of a human hereditary amyloid. Biochem Biophys Res Commun 1988;156:762-8.

11. Soutar AK, Hawkins PN, Vigushin DM, et al. Apolipoprotein AI mutation Arg-60 causes autosomal dominant amyloidosis. Proc Natl Acad Sci U S A 1992;89:7389-93.

12. Pepys MB, Hawkins PN, Booth DR, et al. Human lysozyme gene mutations cause hereditary systemic amyloidosis. Nature 1993;362:553-7.

13. Benson MD, Liepnieks J, Uemichi T, Wheeler G, Correa R. Hereditary renal amyloidosis associated with a mutant fibrinogen $\alpha$-chain. Nat Genet 1993;3:252-5.

14. Holmgren G, Costa PM, Andersson C, et al. Geographical distribution of TTR met 30 carriers in northern Sweden: discrepancy between carrier frequency and prevalence rate. J Med Genet 1994;31:351-4.

15. Kyle RA, Gertz MA. Primary systemic amyloidosis: clinical and laboratory features in 474 cases. Semin Hematol 1995;32:45-59.

16. Booth DR, Tan SY, Hawkins PN, Pepys MB, Frustaci A. A novel variant of transthyretin, 59 $9^{\text {Thr-Lys }}$, associated with autosomal dominant cardiac amyloidosis in an Italian family. Circulation 1995;91:962-7.

17. Gillmore JD, Booth DR, Rela M, et al. Curative hepatorenal transplantation in systemic amyloidosis caused by the Glu526Val fibrinogen $\alpha$-chain variant in an English family. QJM 2000;93:269-75.

18. Puchtler $\mathrm{H}$, Sweat F, Levine $M$. On the binding of Congo red by amyloid. J Histochem Cytochem 1962;10:355-64.

19. Tennent GA. Isolation and characterization of amyloid fibrils from tissue. In: Wetzel R, ed. Methods in enzymology. Vol. 309. Amyloid, prions, and other protein aggregates. San Diego, Calif.: Academic Press, 1999:26 47.

20. Kyle RA, Therneau TM, Rajkumar SV, et al. A long-term study of prognosis in monoclonal gammopathy of undetermined significance. N Engl J Med 2002;346:564-9.

21. Linke RP, Gärtner $H V$, Michels $H$. High-sensitivity diagnosis of $A A$ amyloidosis using Congo red and immunohistochemistry detects missed amyloid deposits. J Histochem Cytochem 1995;43:863-9.

22. Connors LH, Richardson AM, Théberge R, Costello CE. Tabulation of transthyretin (TTR) variants as of $1 / 1 / 2000$. Amyloid 2000;7:54-69. 23. Jacobson DR, Pastore RD, Yaghoubian R, et al. Variant-sequence transthyretin (isoleucine 122) in late-onset cardiac amyloidosis in black Americans. N Engl J Med 1997;336:466-73.

24. Benson MD, Liepnieks JJ, Yazaki M, et al. A new human hereditary amyloidosis: the result of a stop-codon mutation in the apolipoprotein AII gene. Genomics 2001;72:272-7.

25. Uemichi T, Liepnieks JJ, Benson MD. Hereditary renal amyloidosis with a novel variant fibrinogen. J Clin Invest 1994;93:731-6.

26. Uemichi T, Liepnieks JJ, Alexander F, Benson MD. The molecular ba sis of renal amyloidosis in Irish-American and Polish-Canadian kindreds. QJM 1996;89:745-50.

27. Gillmore JD, Booth DR, Madhoo S, Pepys MB, Hawkins PN. Hereditary renal amyloidosis associated with variant lysozyme in a large English family. Nephrol Dial Transplant 1999;14:2639-44.

28. Vigushin DM, Gough J, Allan D, et al. Familial nephropathic systemic amyloidosis caused by apolipoprotein AI variant Arg 26. QJM 1994;87: 149-54.

29. Hamidi Asl L, Liepnieks JJ, Hamidi Asl K, et al. Hereditary amyloid cardiomyopathy caused by a variant apolipoprotein AI. Am J Pathol 1999; 154:221-7.

30. de Sousa MM, Vital C, Ostler D, et al. Apolipoprotein AI and trans thyretin as components of amyloid fibrils in a kindred with apoAI Leul78His amyloidosis. Am J Pathol 2000;156:1911-7.

31. Holmgren G, Ericzon B-G, Groth C-G, et al. Clinical improvement and amyloid regression after liver transplantation in hereditary transthyretin amyloidosis. Lancet 1993;341:1113-6.

Copyright (C) 2002 Massachusetts Medical Society. 